\title{
BLIND SEPARATION AND LOCALIZATION OF DIPOLE SOURCES OF MEG *
}

\author{
Francis H. Y. Chan, Chunqi Chang, Weichao Xu, F. K. Lam, and Paul Kwok \\ Department of Electrical and Electronic Engineering, the University of Hong Kong , Hong Kong. \\ email: fhychan@eee.hku.hk
}

\begin{abstract}
We present a new approach to MEG inverse problem by modeling it into a standard blind source separation problem. In our approach, dipole sources and gain matrix are estimated without any knowledge about the head geometry and conductivity. Given the head model, we can compute dipole locations further. Our matrix pencil method developed before is suitable for this task and is applied in the simulation. Simulation results are presented.
\end{abstract}

key words: Blind source separation, source localization, matrix pencil, magneto-encephalographic.

\section{INTRODUCTION}

Source localization of MEG is very useful and important for studying brain function and for diagnostic purpose. In literature, to estimate source locations dipole model is usually assumed and head model is assumed known. However, head model can never be accurately obtained, and complicated model makes source localization a hard task. Now we propose a different approach to this inverse problem. Since the sources are generated at different location and independently by different neurons, it is reasonable to assume that they are uncorrelated from each other and have different spectra. Under these assumptions, we can use the second order statistics based blind signal separation method we developed before to estimate the source signals and the gain matrix between internal sources and external measurements. In addition, if a head model is available, the source locations can also be estimated from the estimated gain matrix, and the estimation procedure may be much simpler and easier than those estimating source locations directly from MEG measurements.

\section{METHODS}

Consider $L$ dipole sources with fixed or slowly moving locations $\vec{r}_{l}$ and moments $\vec{j}_{l}(t), l=1, \cdots, L$, a sensor located at $\vec{r}_{m}$ measures the magnetic field strength as $b\left(\vec{r}_{m}, t\right)=$ $\sum_{l=1}^{L} \vec{g}\left(\vec{r}_{m}, \vec{r}_{l}\right) \cdot \vec{j}_{l}(t)+n(t)$, where $\vec{g}$ is the gain coefficient and $n(t)$ is the measurement noise. If dipole $l$ has fixed orientation, $\vec{j}_{l}(t)=\left[j_{l, x} j_{l, y} j_{l, z}\right]^{T} j_{l}(t)$; otherwise it is a rotating dipole, $\vec{j}_{l}(t)=\left[j_{l, x}(t) j_{l, y}(t) j_{l, z}(t)\right]^{T}$. Then for both

\footnotetext{
*This research is supported in part by RGC grants.
}

fixed orientation and rotating dipoles, the measurements of $M$ sensors can be written as $B(t)=G \vec{j}(t)+\vec{n}(t)$, which is an additive noise corrupted linear mixture of uncorrelated sources. Then our matrix pencil algorithm for blind source separation can be applied to extract the dipole sources $\vec{j}(t)$ and estimate the gain matrix $G[1]$. Given the head model, the locations can be further estimated through $G$ by applying nonlinear optimization technique.

\section{SIMULATION RESULTS}

The simplest spherically symmetric conductor head model was used in our simulation. Data were simulated for the case of three dipoles randomly located with radius between $50 \mathrm{~mm}$ and $96.9 \mathrm{~mm}$, all with fixed locations and fixed moment orientations. The first source has its main spectrum between $0 \mathrm{~Hz}$ and $100 \mathrm{~Hz}$, the second one has its main spectrum between $12 \mathrm{~Hz}$ and $15 \mathrm{~Hz}$, and the third is a $17 \mathrm{~Hz}$ sinusoid. The data were computed for an array of 39 sensors positioned on the surface of a virtual sphere of radius $111.4 \mathrm{~mm}$. A total of 1000 time samples with sam. pling frequency $1000 \mathrm{~Hz}$ were generated, and then white Gaussian noise was added to obtain an SNR of $10 \mathrm{~dB}$. Our matrix pencil algorithm for blind source separation was used to extract the source signals from the simulated MEG. For the estimated source signals, the signal to interference ratio (SIR) is $13.5 \mathrm{~dB}$, the SNR is $25.1 \mathrm{~dB}$.

\section{CONCLUSIONS}

Blind source separation can be used to extract internal dipole sources, even when the head model is completely unknown. Simulations using our matrix pencil algorithm demonstrate that it works well for MEG source separation. Given the head model, dipole locations can be further estimated. Due to the similarity between MEG and EEG, this technique is equally applicable to EEG. Future works include more accurate simulation and real data processing.

\section{References}

[1] C. Chang, Z. Ding, S. Yau and F. Chan, "A matrixpencil approach to blind separation of non-white signals in white noise", Proc. ICASSP'98, vol. IV, pp.24852488, Seatle, May 1998. 